\section{Características epidemiológicas del síndrome de Guillain-Barré en población chilena: estudio hospitalario en un período de 7 años}

\author{
GABRIEL CEA $^{1}$, PAULA JARA ${ }^{2}$, FERNANDO QUEVEDO $^{3}$
}

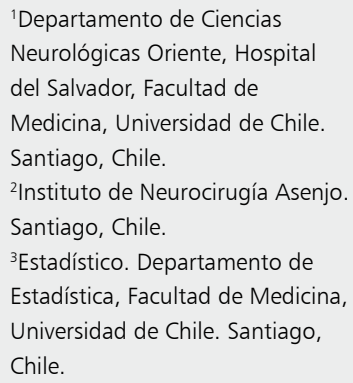

El trabajo fue realizado sin el requerimiento de ayudas o fuente de financiamiento. Los autores no tienen conflicto de intereses que declarar.

Recibido el 6 de abril de 2014, aceptado el 17 de diciembre de 2014.

Correspondencia a:

Gabriel Cea

Servicio de Neurología Hospital

del Salvador

Avenida Salvador 364,

Providencia, Santiago, Chile.

jcea@med.uchile.cl GBS variety was found in 66\% of cases. According to the Hughes' disability score, patients treated with plasmapheresis, showed non-statistically significant better outcomes than those treated with intravenous immunoglobulin. Conclusions: In this group of patients the demyelinated variety of GBS was more common than the axonal type. Although not statistically significant, the better response to plasmapheresis is encouraging and should prompt a controlled study.

(Rev Med Chile 2015; 143: 183-189)

Key words: Epidemiology; Immunoglobulins, intravenous, plasmapheresis; Guillain-Barré Syndrome.

4 1 síndrome de Guillain-Barré (SGB) es una poliradiculopatía aguda que, en un porcentaje alto, se asocia con antecedentes de infecciones digestivas o respiratorias y ocasionalmente con inmunizaciones. Es considerado la causa más importante de parálisis flácida aguda a nivel mundial, con una incidencia entre 0,6 y 4 por 100.000 por año ${ }^{1-3}$. A pesar de su baja incidencia, es una enfermedad que tiene un alto impacto debido a que genera invalidez transitoria, hospitalizaciones prolongadas con cuidados intensivos y procedimientos de alto costo, discapacidad secuelar hasta en $20 \%$ de los casos y mortalidad entre $2 \%-10 \%{ }^{4,5}$. El SGB afecta a todos los grupos etarios, pero es infrecuente en la infancia, y es una de las pocas enfermedades autoinmunes que afecta con mayor frecuencia a hombres ${ }^{6}$. Desde la descripción realizada por Guillain, Barré y Strohl ${ }^{7}$, se han descrito al menos 6 variedades de SGB: la polineuropatía desmielinizante aguda (AIDP), la polineuropatía axonal aguda (AMAN), la polineuropatía axonal sensitivo motora aguda (AMSAN), la polineuropatía axonal sensitiva aguda (ASAN), el síndrome de Miller-Fisher (MF) y la pandisautonomía aguda ${ }^{8,9}$.

La mayoría de los estudios que han caracterizado el SGB proceden de Estados Unidos, Europa, Japón, China o Australia. Estos estudios muestran que la incidencia de los diferentes subtipos de SGB varía considerablemente entre 
los distintos continentes y grupos étnicos. En países como Estados Unidos, Canadá y Australia aproximadamente entre $85 \%$ y $90 \%$ de los casos corresponden a AIDP y $5 \%$ corresponde a las variedades axonales ${ }^{4,6}$. En cambio en China el $70 \%$ de los casos corresponden a la variante axonal AMAN y menos de $25 \%$ a AIDP o a otras variantes $^{1,2}$. En América Latina la información es escasa y con datos fragmentarios, en su mayoría de tipo retrospectivo, con publicaciones que oscilan entre una mayor incidencia de formas axonales ${ }^{1} y$ mayor incidencia de la formas desmielinizantes ${ }^{10}$. En Chile, hasta donde sabemos, no existe información sobre las características epidemiológicas del SGB. El propósito de este estudio es entregar información que contribuya a la caracterización del SGB en Chile.

\section{Pacientes y Método}

Se realizó un análisis retrospectivo de las fichas de los pacientes ingresados al Hospital del Salvador con el diagnóstico de SGB, durante el período de enero de 2003 a diciembre de 2009. Para esto, se hizo una búsqueda en la base de datos de la Unidad de Análisis Clínico del Hospital Salvador, usando la clasificación internacional de enfermedades décima versión (CIE-10). Se utilizaron los siguientes grupos diagnósticos: G61.0 (síndrome de Guillain Barré), G61.8 (polineuropatías, otras patologías inflamatorias) y G62.8 (Otras polineuropatía especificadas). Adicionalmente, se revisaron todas las fichas de los pacientes que se controlaron en el Servicio de Neurología con diagnóstico de SGB durante ese período.

Se identificó un total de 62 fichas clínicas, de las cuales 41 correspondían a pacientes con SGB. En las 21 restantes, 7 casos correspondían a otro tipo de neuropatías, 8 casos eran polineuropatías inflamatorias desmielinizantes crónicas (CIDP) y 6 fichas no contenían datos suficientes para realizar el análisis de los pacientes. La revisión de fichas de pacientes con diagnóstico de SGB en control en el policlínico del Hospital del Salvador, no detectó pacientes que no hubiesen sido pesquisados en la base de datos de nuestro hospital.

Las fichas clínicas fueron analizadas, en base a un esquema de extracción de información previamente establecida que incluía: datos demográficos y epidemiológicos, manifestaciones clínicas y estudios electrofisiológicos (electromiografía, velocidad de conducción motora y sensitiva, onda F y reflejo H) y resultados de laboratorio. Los pacientes fueron clasificados en las distintas variantes de SGB en base a los criterios clínicos y electrofisiológicos aceptados en la literatura ${ }^{8,9}$. Para la determinación de la severidad del cuadro clínico se utilizó la escala de discapacidad de Hughes ${ }^{11}$. Para obtener una medida aproximada de la incidencia de SGB se usaron los datos de la Cuenta Anual del Hospital Salvador de los años analizados. Esta cuenta incluye la población total mayor de 15 años a la cual el hospital entrega cobertura de atención (http://www.hsalvador.cl/cuentas-publicas-hds/). Debido al elevado costo del tratamiento del SGB y la necesidad de cuidados intensivos en una alta proporción de pacientes, es muy poco probable que un paciente de la población asignada consulte en otro centro público o privado y si lo hace es altamente probable que ese paciente sea derivado para su tratamiento a nuestro centro. El uso de las fichas de los pacientes hospitalizados es la fuente más usada en los estudios de incidencia de esta patología, dado que por los síntomas de esta enfermedad el paciente consulta, y al hacerlo es hospitalizado para observación ${ }^{12}$. Se usó el test de Mann-Whitney para comparar el resultado del tratamiento entre los grupos de tratamiento. Este trabajo se realizó cumpliendo las normas éticas internacionalmente exigidas cuando compromete seres humanos y fue aprobado por el Comité de Ética Científico del Servicio de Salud Metropolitano Oriente de Santiago.

\section{Resultados}

Cuarenta y un pacientes fueron analizados, de los cuales 30 correspondían a pacientes hombres y 11 a mujeres, con una relación de 2,7:1. El promedio de edad fue de 51,6 años (rango 17-81), con mayor número de pacientes en los grupos etáreos entre 36-65 años (Tabla 1 y Figura 1). En base al número de casos en cada año se obtuvo una medida aproximada de la incidencia que fluctuó entre 1,0 y 1,7 por 100.000 habitantes. De acuerdo a los hallazgos clínicos y electrofisiológicos, se identificaron las siguientes variantes o subtipos de SGB: AIDP 27 pacientes, AMAN 7 pacientes, AMSAN 2 pacientes, ASAN 2 pacientes y MF 3 pacientes (Tabla 1). Desde el inicio de los síntomas hasta la 
Síndrome de Guillain-Barré en Chile: estudio hospitalario - G. Cea et al

Tabla 1. Características clínicas y epidemiológicas: promedio (rango)

\begin{tabular}{|c|c|c|c|c|c|}
\hline & AIDPa & AMAN $^{b}$ & AMSAN $^{c}$ & ASAN $^{d}$ & MFe \\
\hline Pacientes (número) & 27 & 7 & 2 & 2 & 3 \\
\hline Edad (años) & $50,3(17-81)$ & $48,6(20-62)$ & $57,5(42-73)$ & $51(42-60)$ & $69,3(60-79)$ \\
\hline Hombres/Mujeres & $19 / 8$ & $6 / 1$ & $1 / 1$ & $2 / 0$ & $2 / 1$ \\
\hline Días previos a hospitalización & $7,9(1-30)$ & $10,8(2-25)$ & $18,5(2-35)$ & $20(10-30)$ & $6(3-10)$ \\
\hline Días hospitalización & $18,7(5-42)$ & $20,3(14-35)$ & $47(41-53)$ & $32,5(16-49)$ & $23(14-35)$ \\
\hline \multicolumn{6}{|l|}{ Infecciones previas } \\
\hline Gastrointestinal & 7 & 4 & ---- & ---- & ---- \\
\hline Respiratoria & 5 & ---- & 1 & ---- & 1 \\
\hline Otras infecciones & 3 & ---- & ---- & ---- & ---- \\
\hline Inmunizaciones & 1 & ---- & ---- & ---- & ---- \\
\hline
\end{tabular}

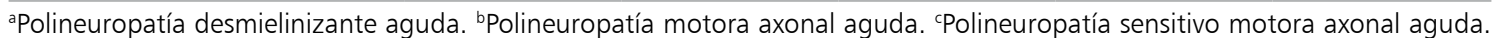
dPolineuropatía axonal sensitiva aguda. eSíndrome de Miller-Fisher.

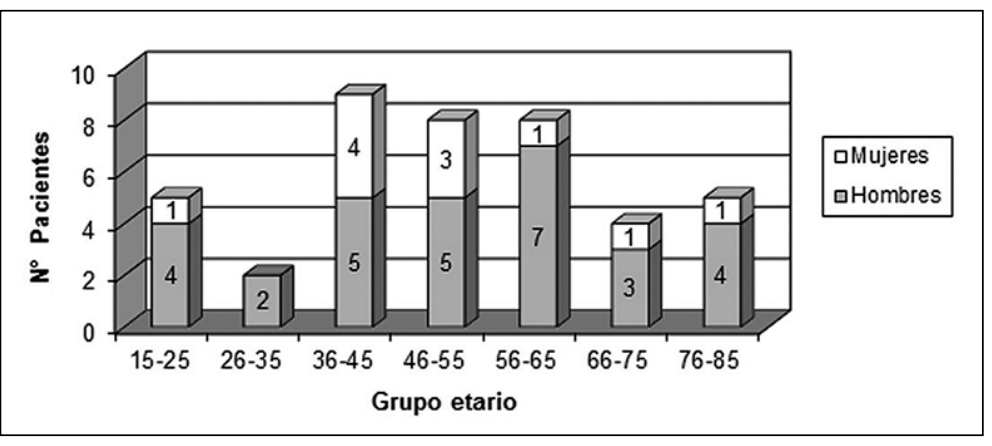

Figura 1. Distribución por edad y sexo en pacientes con Síndrome de Guillain -Barré ingresados entre los años 20032009. hospitalización transcurrieron en promedio 9,2 días (rango 2-35), siendo las variedades AMSAN y ASAN las con mayor retardo en su hospitalización y diagnóstico.

La mayoría de los casos se presentaron en los meses de verano e invierno (Figura 2). Veintiún pacientes $(51,2 \%)$ habían presentado cuadros infecciosos en las 2 o 3 semanas anteriores al diagnóstico, principalmente gastrointestinales o respiratorios, sin que esto determinara diferencias importantes entre las distintas variedades clínicas (Tabla 1). Otras patologías concomitantes incluyeron: diabetes mellitus en 6 pacientes, hipotiroidismo en 1 paciente e infección por el virus de la inmunnodeficiencia humana (VIH) en un paciente con tratamiento antirretroviral (TARV) por 5 años. Un paciente tenía el antecedente de SGB hace 8 años y otro de MF hace 2 años. El estudio de líquido cefalorraquídeo se realizó en 40 pacientes. En 21 pacientes (52,5\%) este mostró disociación albumino-citológica y en 19 fue normal. En promedio, el momento de la punción lumbar en los pacientes con líquido disociado fue a los 15,2 días (2-42 días) y a los 10,8 días (3-30 días) en los con líquido normal, similar a otras series ${ }^{13}$.

$\mathrm{Al}$ ingreso, los hallazgos clínicos más frecuentes fueron: tetraparesia en 27 pacientes; debilidad facial de diferente severidad en 17 pacientes; hiporreflexia o arreflexia osteotendínea en la totalidad de los pacientes y síntomas sensitivos en 14 pacientes. En la Figura 3 se muestra la distribución, al ingreso y al egreso, del grado de discapacidad de acuerdo a la escala de Hughes en la totalidad del grupo analizado. $\mathrm{Al}$ ingreso, 11 pacientes con AIDP y los 7 con la variedad AMAN no podían caminar, 5 con AIDP y uno con variedad ASAN podían caminar con ayuda, 5 AIDP eran capaces de caminar pero no de correr y otros 6 pacientes 

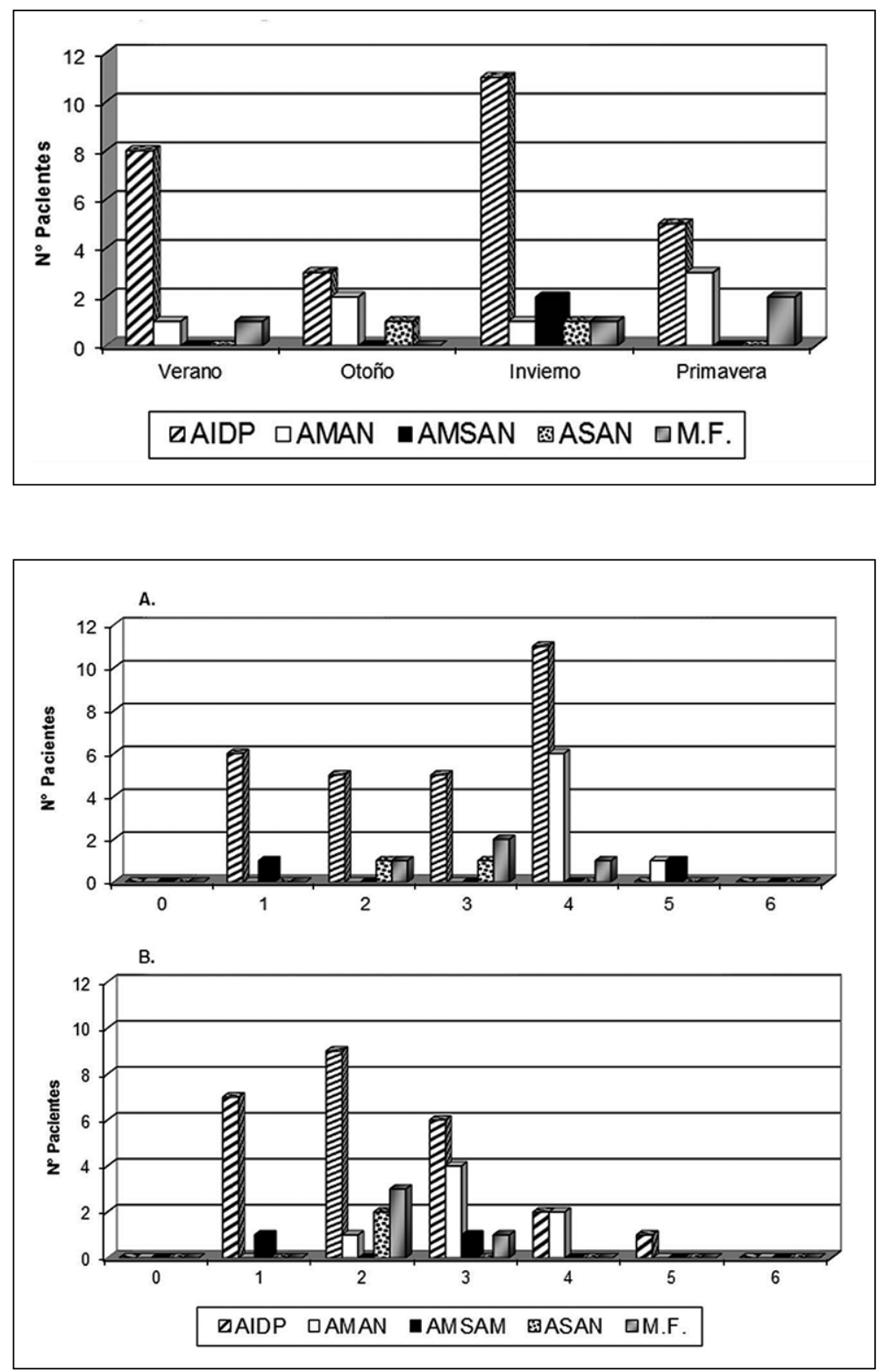

Figura 2. Variaciones estacionales del Síndrome de Guillain-Barré de los pacientes ingresados entre los años 2003-2009.

Figura 3. Severidad de la discapacidad en los pacientes con Síndrome de Guillain -Barré al ingreso (A) y egreso (B) durante los años 2003-2009 según escala de discapacidad de Hughes.

Escala de discapacidad de Hughes para SGB: 0 = sano, 1 = síntomas menores, capaz de correr, 2 = capaz de caminar 10 metros sin asistencia pero incapaz de correr, 3 = capaz de caminar 10 metros con ayuda, 4 = postrado o en silla de rueda, 5 = requiere ventilación mecánica al menos parte del día, 6 = muerto.

con AIDP y uno con la variedad ASAN tenían síntomas menores. Tres pacientes, correspondientes a la variedad AMAN, AMSAN y AIDP, requirieron ventilación mecánica. Al egreso la mayoría de los pacientes con subtipo AIDP (59,2\%) presentaban discapacidad menor, $22,2 \%$ requirió ayuda para caminar y sólo 3,7\% estaba postrado. En cambio, en el subtipo AMAN el grado de discapacidad fue mayor, $57 \%$ de pacientes requirió ayuda para caminar y $28,5 \%$ estuvo postrado. La mayoría de los pacientes con MF fue capaz de caminar pero no de correr. Un paciente con la variedad AMSAN presentó déficit menores y el segundo requirió ayuda para caminar.

Nueve pacientes, que en promedio tenían una discapacidad de 3 en escala de Hughes (ver leyenda en Figura 3), recibieron inmunoglobulinas endovenosas, en dosis habituales de $2 \mathrm{~g} / \mathrm{Kg}$ administrada en 4 ó 5 días, y 17 pacientes que tenían en promedio una discapacidad de 3,5, 
fueron sometidos a plasmaféresis en $40 \mathrm{~mL} / \mathrm{kg}$ de volumen de plasma por sesión, en 3 o 5 sesiones, como es también habitual. Tres pacientes fueron sometidos a ambos tratamientos. Por otra parte, en 9 pacientes con discapacidad máxima de 3 y un promedio de discapacidad de 2 , no se consideró necesario ninguno de estos procedimientos debido a la estabilización de sus síntomas, y sólo recibieron medidas de apoyo y rehabilitación. En términos globales la mayoría de los pacientes tratados experimentó una mejoría entre su condición de ingreso y egreso, de acuerdo a la escala de discapacidad de Hughes (Figura 3). El 68,4\% de los tratados con plasmaféresis experimentó una mejoría de un punto o más en la escala de discapacidad de Hughes, comparado con $44,4 \%$ de los tratados con inmunoglobulina endovenosa. Esta diferencia en la disminución en la escala de discapacidad no presenta diferencia estadísticamente significativa entre los tratamientos. En el grupo que sólo recibió medidas de apoyo, también se observó una mejoría de un punto o más de la escala de Hughes en $44,4 \%$ de los pacientes. No hubo pacientes fallecidos en nuestra serie como resultado de la enfermedad o complicaciones del tratamiento.

\section{Discusión}

Hasta donde sabemos, no existe información de la incidencia del SGB en Chile ni en la mayoría de los países de America Latina. Las cifras de incidencia provienen de la literatura norteamericana, europea o japonesa. Teniendo en cuenta la población a la que se da cobertura, nuestra serie establece una medida aproximada de la incidencia que va desde 1,0 a 1,7 x 100.000 en los siete años analizados. Este método aproximado, a partir de la revisión de fichas hospitalarias de hospitales a los cuales es posible asignarle una población definible, es usado en numerosas publicaciones reportando incidencia de $\mathrm{SGB}^{12}$. Nuestra incidencia estimativa está en acuerdo con cifras proporcionada por la literatura mundial, como se observa en revisiones extensas sobre la literatura, con una incidencia entre 1,1 y 1,8 por 100.000 casos anuales ${ }^{2}$ y entre 0,81 y 1,89 por 100.000 casos anuales ${ }^{12}$.

El SGB es uno de los pocos trastornos autoinmunes donde la incidencia es mayor en hombres que en mujeres. En nuestra serie esta relación fue de 2,7:1, que es algo mayor que lo comunicado en la literatura (alrededor de 1,5:1) 6,13-15. Con respecto a los rangos de edad, nuestros casos se presentaron preferentemente entre los 36-65 años, con una disminución en la edades menores y en la población mayor, no observándose un aumento hacia las edades extremas como se ha descrito en otras series ${ }^{14,16,17}$.

En nuestra serie la variedad AIDP fue más común (27 casos de 41, 65,9\%) que las variedades axonales (11 pacientes, 26,7\%). Los estudios realizados en Norteamérica, Europa y Australia describen a la variante AIDP con una frecuencia entre $85 \%$ y $90 \%$ y con sólo $5 \%$ para la variantes axonal ${ }^{1,2}$. Por otra parte, en China, predominan las variantes axonales, siendo la variedad AMAN la que alcanza una frecuencia de $70 \%$ y menos de $25 \%$ para la variedad AIDP y otros subtipos ${ }^{1,2}$. Esto pone a nuestra serie en una situación similar a las series europeas, norteamericanas o japonesas. No obstante, los estudios latinoamericanos existentes revelan cifras contradictorias. Por ejemplo, estudios en niños realizados en México revelan una predominancia de las variedades axonales, tanto en series prospectivas ${ }^{1}$ como en estudios de autopsias ${ }^{1,18}$. Sin embargo, un estudio realizado en Argentina, en población pediátrica, la variedad AIDP es también dominante, alcanzando $70,5 \%{ }^{10}$.

La mayoría de los estudios sobre el SGB reportan el antecedente de enfermedades infecciosas, usualmente entre 2 y 4 semanas previas al inicio del cuadro, con cifras que van entre $40 \%-70 \%$ de los $\operatorname{casos}^{2,3,6}$. En nuestra serie, el $51 \%$ presentaba antecedentes de infección, cifra que concuerda con la literatura. De estos 52,3\% presentó infecciones gastrointestinales y $33 \%$ infecciones respiratorias, lo que difiere de la literatura europea o norteamericana, donde la proporción es inversa, reportándose 22\%-53\% para el tracto respiratorio y 6\%-26\% para el tracto gastrointestinal ${ }^{6,16,19}$. La presentación del SGB en la literatura internacional es esporádica, sin encontrarse descripción de variación estacional estadísticamente significativa con excepción de las áreas rurales en el norte de China donde existe un considerable incremento de casos de tipo axonal en los meses de verano, asociado a infecciones con Campilobacter Jejuni $i^{2,6}$. En nuestro estudio, encontramos variaciones estacionales marcadas, con la mayoría de los casos presentaándose en invierno y verano. En las patologías concomitantes hubo 6 casos de diabetes 
mellitus y uno de hipotiroidismo. En el paciente con VIH creemos que la asociación es fortuita dado que este estaba con TARV desde hace 5 años. Los casos de SGB asociados al VIH generalmente se describen en el momento de la seroconversión $\mathrm{y}$ son de rara incidencia ${ }^{20}$.

Los estudios comparativos revelan que no hay diferencia entre el uso de plasmaféresis e inmunoglobulina, excepto que la plasmaféresis tendría mayor incidencia de efectos secundarios severos $^{21-26}$. Nuestra serie muestra que la plasmaféresis tuvo una tendencia a presentar mejores resultados, si bien no significativa, tanto en el paciente con la variedad desmielinizante, así como en las variedades axonales según la escala de Hughes al momento del alta. Esto nos parece interesante, porque una tendencia a un efecto mejor no significativo ha sido descrita en un estudio comparativo japonés ${ }^{26}$. Esto podría justificar un estudio prospectivo controlado sobre tratamiento en población chilena.

En resumen, este estudio retrospectivo muestra algunas diferencias en el comportamiento de SGB en la población chilena, con las descritas en otras poblaciones. Debido a las limitaciones metodológicas de este trabajo, un estudio prospectivo clínico epidemiológico más amplio es necesario para extraer conclusiones mas confiables respecto del SGB en Chile.

Agradecimientos: A Evelyn Villalón, Jefa del Departamento de Información y Control de Gestión del Hospital del Salvador, por su ayuda en la búsqueda sistemática de las fichas clínicas utilizadas en el estudio.

\section{Referencias}

1. Nachamkin I, Arzarte Barbosa P, Barbosa PA, Ung H, Ung $\mathrm{H}$, Lobato $\mathrm{C}$, et al. Patterns of Guillain-Barre syndrome in children: results from a Mexican population. Neurology. 2007; 69 (17): 1665-71.

2. McGrogan A, Madle GC, Seaman HE, de Vries CS. The epidemiology of Guillain-Barre syndrome worldwide. A systematic literature review. Neuroepidemiology. 2009; 32 (2): 150-63.

3. Hughes RAC, Cornblath DR. Guillain-Barre syndrome. Lancet. 2005; 366 (9497): 1653-66.

4. Van Doorn PA, Ruts L, Jacobs BC. Clinical features, pathogenesis, and treatment of Guillain-Barre syndro- me. Lancet Neurol. 2008; 7 (10): 939-50.

5. Cheng Q, Jiang GX, Press R, Andersson M, Ekstedt B, Vrethem M, et al. Clinical epidemiology of Guillain-Barre syndrome in adults in Sweden 1996-97: a prospective study. Eur J Neurol. 2000; 7 (6): 685-92.

6. Govoni V, Granieri E. Epidemiology of the GuillainBarre syndrome. Curr Opin Neurol. 2001; 14 (5): 60513.

7. Guillain G, Barre J, Strohl A. Sur un syndrome de radiculo-nevrite avec hyperalbuminose du liquid cephalorachidien sans reaction cellulaire. Remarques sur les caracteres cliniques et graphiques desreflexes tendineux. Bull Soc Med Hop Paris. 1916; 28: 1462-70.

8. Van der Meché FG, Van Doorn PA, Meulstee J, Jennekens FG. Diagnostic and classification criteria for the Guillain-Barre syndrome. Eur Neurol. 2001; 45 (3): 133-9.

9. Oh SJ, LaGanke C, Claussen GC. Sensory Guillain-Barre syndrome. Neurology. 2001; 56 (1): 82-6.

10. Paradiso G, Tripoli J, Galicchio S, Fejerman N. Epidemiological, clinical, and electrodiagnostic findings in childhood Guillain-Barre syndrome: a reappraisal. Ann Neurol. 1999; 46 (5): 701-7.

11. Hughes RA, Newsom-Davis JM, Perkin GD, Pierce JM. Controlled trial prednisolone in acute polyneuropathy. Lancet. 1978; 2 (8093): 750-3.

12. Sejvar JJ, Baughman AL, Wise M, Morgan OW. Population incidence of Guillain-Barr? syndrome: A systematic review and meta-analysis. Neuroepidemiology. 2011. p. 123-33.

13. Van der Meché FG, van Doorn PA. Guillain-Barre syndrome and chronic inflammatory demyelinating polyneuropathy: immune mechanisms and update on current therapies. Ann Neurol. 1995; 37 Suppl 1: S14-31.

14. Jiang GX, Cheng Q, Link H, de Pedro-Cuesta J. Epidemiological features of Guillain-Barre syndrome in Sweden, 1978-93. J Neurol Neurosurg Psychiatry. 1997; 62 (5): 447-53.

15. A prospective study on the incidence and prognosis of Guillain-Barre syndrome in Emilia-Romagna region, Italy (1992-1993). Emilia-Romagna Study Group on Clinical and Epidemiological Problems in Neurology. Neurology. 1997; 48 (1): 214-21.

16. Bogliun G, Beghi E. Incidence and clinical features of acute inflammatory polyradiculoneuropathy in Lombardy, Italy, 1996. Acta Neurol Scand. 2004; 110 (2): 100-6.

17. Rees JH, Thompson RD, Smeeton NC, Hughes RA. Epidemiological study of Guillain-Barre syndrome in south east England. J Neurol Neurosurg Psychiatry. 1998; 64 (1): 74-7. 
18. Ramos-Álvarez M, Bessudo L, Sabin AB. Paralytic syndromes associated with noninflammatory cytoplasmic or nuclear neuronopathy. Acute paralytic disease in Mexican children, neuropathologically distinguishable from Landry-Guillain-Barre syndrome. JAMA. 1969; 207 (8): 1481-92.

19. Koga M, Yuki N, Hirata K. Antecedent symptoms in Guillain-Barre syndrome: an important indicator for clinical and serological subgroups. Acta Neurol Scand. 2001; 103 (5): 278-87.

20. Fuller GN, Jacobs JM, Guiloff RJ. Nature and incidence of peripheral nerve syndromes in HIV infection. J Neurol Neurosurg Psychiatry. 1993; 56 (4): 372-81.

21. Plasma exchange in Guillain-Barre syndrome: one-year follow-up. French Cooperative Group on Plasma Exchange in Guillain-Barre Syndrome. Ann Neurol. 1992; 32 (1): 94-7.

22. Van der Meché FG, Schmitz PI. A randomized trial comparing intravenous immune globulin and plasma exchange in Guillain-Barre syndrome. Dutch Guillain-
Barre Study Group. N Engl J Med. 1992; 326 (17): 1123 9.

23. Appropriate number of plasma exchanges in GuillainBarre syndrome. The French Cooperative Group on Plasma Exchange in Guillain-Barre Syndrome. Ann Neurol. 1997; 41 (3): 298-306.

24. Randomised trial of plasma exchange, intravenous immunoglobulin, and combined treatments in GuillainBarre syndrome. Plasma Exchange/Sandoglobulin Guillain-Barre Syndrome Trial Group. Lancet. 1997; 349 (9047): 225-30.

25. Diener HC, Haupt WF, Kloss TM, Rosenow F, Philipp $\mathrm{T}$, Koeppen S, et al. A preliminary, randomized, multicenter study comparing intravenous immunoglobulin, plasma exchange, and immune adsorption in GuillainBarre syndrome. Eur Neurol. 2001; 46 (2): 107-9.

26. Hughes RAC, Swan A V, Raphaël J-C, Annane D, van Koningsveld R, van Doorn PA. Immunotherapy for Guillain-Barre syndrome: a systematic review. Brain. 2007; 130 (Pt 9): 2245-57. 